\title{
ENERgy EFFiCiENCY Cross LAYER Protocol FOR WIRELESS MESH NETWORK
}

\author{
Sarra MAMECHAOUI ${ }^{1}$, Fedoua DIDI $^{1}$ and Guy PUJOLLE ${ }^{2}$ \\ ${ }^{1}$ STIC Laboratory, Abou Bekr Belkaid University Tlemcen, Algeria \\ ${ }^{2}$ Pierre et Marie Curie University Paris 6, France
}

\begin{abstract}
Wireless mesh network (WMN) is a novel emerging technology that will change the world more effectively and efficiently. It is regarded as a highly promising technology being increasingly important in mobile wireless networks of the future generation. In this paper, we consider energy management for wireless mesh networks from a point of view that started recently to attract the attention means the conservation of energy for operational and the environment reasons which is known as the Green Networking. This paper discusses different routing protocols to establish a protocol which considers energy efficiency. The existing protocols are compared using the basic functions of routing and the suggest protocol is designed to overcome some of their shortcomings. We are focusing on the conception of the cross-layer routing protocol that is implemented in TDMA (Time Division Multiple Access) wireless mesh networks based MAC protocol.
\end{abstract}

\section{KEYWORDS}

Wireless Mesh Network, Routing protocols, energy consumption, TDMA, NS 2.

\section{INTRODUCTION}

Wireless mesh networks (WMNs) are an emerging trend in wireless communication more flexible, reliable and effective than the conventional wireless LANs (WLAN). Wireless mesh network and mobile ad hoc network use the same key concept communication between nodes over multiple wireless hops on a meshed network graph. Nevertheless, they highlight various aspects. Mobile ad hoc networks (MANET) have an academic background and concentrate on end users, mobility and ad hoc capabilities. WMNs have a background in business and focus mostly on static devices (often infrastructure), reliability, network capacity and practical deployment [1].

WMNs architecture can be categorized into three principal groups on the basis of the node functionality [2]:

- Infrastructure/Backbone WMNs: This type of WMNs architecture is comprises of mesh routers which form an infrastructure for clients that are connecting to them.

- Client WMNs: client nodes form the real network to carry out the routing and the configuration as well as providing end-user applications to clients. Therefore, a mesh router is not necessary for such networks. 
- Hybrid WMNs: This architecture is the combination of infrastructure and client WMNs architecture as shown in Figure. 1.

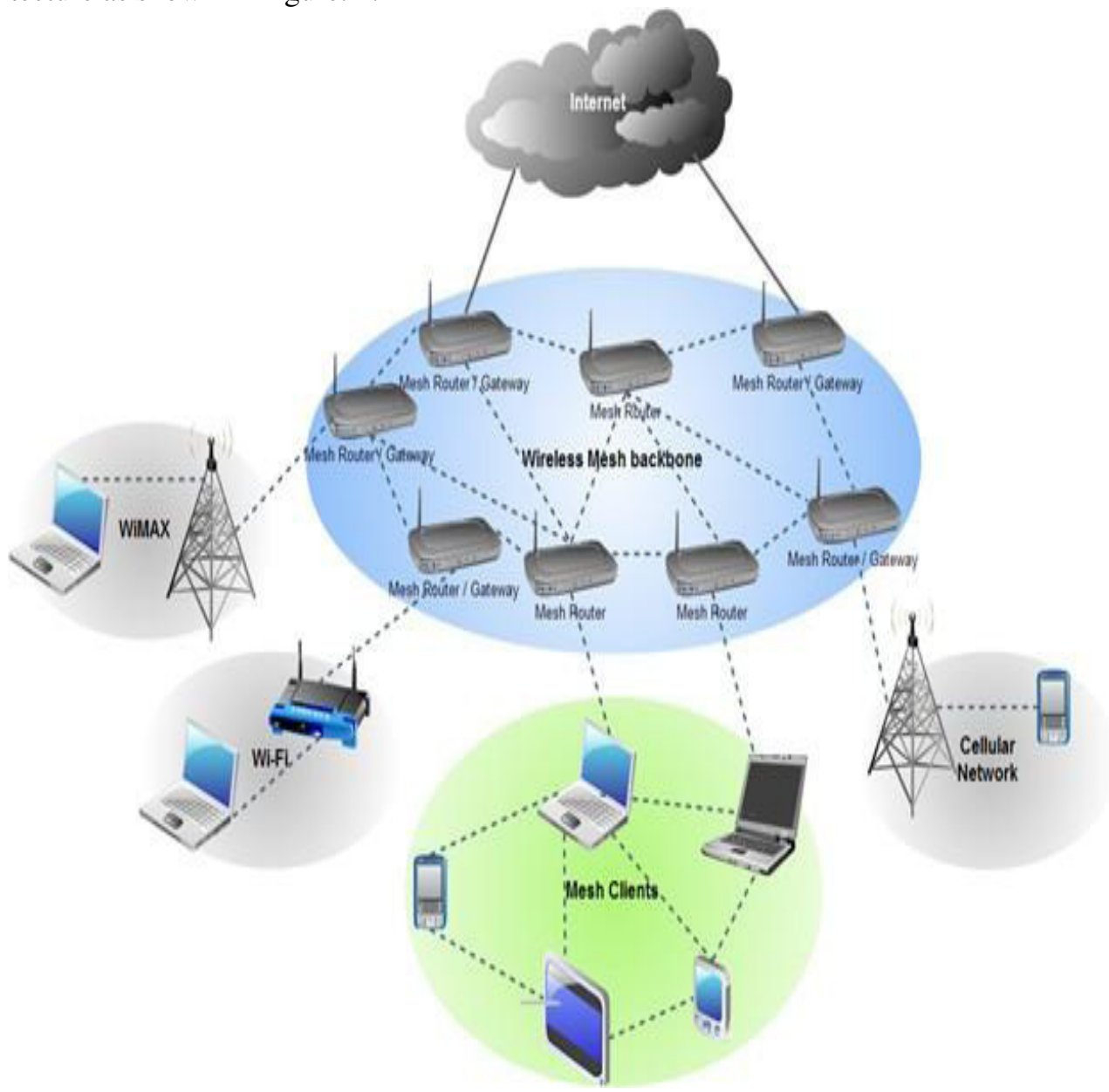

Figure 1. Hybrid WMNs

In the majority of WMNs most of nodes are stationary or have a minimum mobility and do not rely on batteries. Therefore, the developing of routing algorithms is to enhance network throughput or performance independent transfers, instead of dealing with mobility or minimizing power consumption. However in this paper, we consider energy management for wireless mesh networks from a point of view that started recently to attract the attention means the conservation of energy for operational and the environment reasons which is known as the Green Networking. This aims to reduce the environmental footprint, economic and social information and communication technology (ICT). Green networking solution provides global environmental network architectures to improve energy efficiency, performance and reduce the cost savings, it also allows companies to gain competitive advantage and maintain a sustainable environment. The recent studies have estimated that the accounts of the ICT industry represent approximately $2 \%$ of global $\mathrm{CO}_{2}$ emissions. In detail, with a focus on telecommunications networks, these are estimated to produce about $0.6 \%$ of global $\mathrm{CO}_{2}$ emissions [3].

To solve this problem, it is essential that we aim to improve and sustain performance of ICT and minimize their energy consumption and carbon footprint. Even if the functions of routing protocols are plausible, they are always facing challenges in terms of energy efficiency. So there is requiring for a routing protocol which will be energy efficient as well as scalable. This paper 
International Journal of Computer Networks \& Communications (IJCNC) Vol.6, No.3, May 2014

reviews different routing protocols focusing on the request of energy efficiency. The routing protocols are compared using the basic features of routing and the proposed protocol is designed to overcome some of their shortcomings, specifically energy efficiency.

The rest of the paper is structured as follows: Section 2 explores the existing routing protocols. Section 3 discusses different routing protocols focusing on the request of energy efficiency. Section 4 presents the model of a Time Division Multiple Access (TDMA) protocol to schedule flows of traffic in slots and finally Section 5 concludes the paper.

\section{Routing Protocols}

Generally, there are two families of routing protocols [4]: routing protocols called proactive, anticipating the demand for packet routing and reactive routing protocols that respond to the request. Between these two families, a new approach is emerging: it is the protocols called "hybrid" which are inspired by both proactive and reactive protocols.

The basic functionality of multi-hop wireless ad hoc wireless networking, as well as wireless mesh networks is the routing capacity. The routing protocols anticipate the required paths via a WMN, such that the nodes may communicate in good or optimal paths of multiple wireless hops. From the moment that WMNs have common features with the ad hoc wireless networks, routing protocols developed for the MANET can be applied to WMNs [1].Such as Microsoft Mesh Networks [5] are designed on the basis of Dynamic Source Routing (DSR) [6], and for example [7] use ad hoc On-demand Distance Vector (AODV) routing [8] . Occasionally, the basic concepts of routing protocols that exist have been extended to meet the specific needs of wireless mesh networks, for example, with radio aware routing metrics in IEEE 802.11s WLAN mesh network standardization.

The presentation of the following routing protocols is far from being exhaustive. There are many others, but this selection covers most standard protocols and is studied. In preoccupations of brevity, a global vision of protocols is given, with the essential features, but only used to for comparison as a support or base for the rest.

\subsection{Ad hoc On-demand Distance Vector Routing Protocol (AODV)}

AODV [8], is a highly popular routing protocol for MANET. It is a reactive routing protocol. Routes are established on demand, and only the active channels are kept. This protocol implements various operations to achieve and maintain routing: local connectivity management, discovery of routes and maintenance of routes. AODV uses a simple request-response (RREQs RREP) mechanism for route discovery. It can use hello messages on connectivity and signal link breaks in active routes with error messages. Each of this routing information has a delay associated with it, and a sequence number. The use of sequence numbers to detect outdated data, so that only the information of the most common path used is available. It ensures freedom of routing loops and prevents problems known from classical distance vector protocols, such as "counting to infinity". Each time a node wants to send packets to destination it broadcasts RREQs, the source node uses a research technique in the ring expansion. When a destination or an intermediate node using a route error (RERR) is sent to the source node assigned. When the source node receives (RERR), it can revive route if the route is still needed. Neighborhood information is obtained from emission of Hello packet. This helps to reduce the overhead of routing, but introduces a certain initial latency because of the configuration of the route on demand. There are various implementations available, for instance, SEE-AODV [9] which is scalable and more energy efficient than existing Ad hoc on Demand Distance (AODV) routing protocol in wireless mesh networks. Other example, The PO-AODV [10] means Power 
International Journal of Computer Networks \& Communications (IJCNC) Vol.6, No.3, May 2014

Optimized Ad hoc On demand Distance Vector routing protocol that regards the remaining energy of the nodes on the path for finding the optimal path from source to destination.

\subsection{Ad hoc On-demand Multipath Distance Vector Routing Protocol (AOMDV)}

Multipath variant of AODV called AOMDV [11]. Here, multiple disjoint paths are maintained for each destination. When the primary path fails an alternate path is available instantaneously. This eliminates the route rediscovery overhead. The key idea of AOMDV protocol is to compute multiple paths during route discovery. It consists of two components: updating the route for establishing and maintaining the node and the distributed protocol to discover link-disjoint paths. In AOMDV each RREQ, respectively RREP arriving at a node potentially is defining an alternate path to the source or destination.

When a node S floods a RREQ packet in the network, each RREQ destined for the node I via a another neighbor of S, or S itself, defines a node disjoint path from I to S. In AOMDV this is used at the intermediate nodes. Duplicate copies of a RREQ are not immediately discarded. Each packet is examined to see if it provides a node-disjoint path to the source. For node disjoint paths all RREQs need to arrive via different neighbors of the source. At the destination a slightly different approach is used, the paths determined there are link disjoint, not node-disjoint. In order to do this, the destination responds up to k copies of the RREQ. The RREQs only need to arrive via unique neighbors.

\subsection{Destination- Sequenced Distance-Vector (DSDV)}

Destination-Sequenced Distance-Vector (DSDV) [12], is a proactive routing algorithm. It is based on distance vector algorithm which allows limiting the exchange of control messages topology only neighbors of node. The DSDV protocol uses the diffusion properties to transmit routing information. Indeed, the great advantage of the broadcast is a frame sent by a station is heard by all its neighbors. Periodically, each station broadcasts its entire routing table followed by a number to date information. This number is called the sequence number. From two sequence numbers, it is possible to determine what information is the most recent. The routing table of a node contains information related to each route. Upon reception of this information, neighbors update their routing table following a specific pattern. Every entry of the routing table is updated only if the received information is more new or if it is the same age but has a smaller number of nodes. Eventually, the DSDV protocol provides for each destination, the route that has the smallest number of nodes. To be a full routing protocol, the DSDV protocol must maintain the status of the paths. For this, nodes detect the link failures. Each node transmits periodically its routing information to all its neighbors. A node detecting a break, will broadcast a packet containing all the destinations that can be reached through this link. Any node receiving such a packet propagates immediately to inform the faster the topology change. One problem with this algorithm is that it reacts too slowly to bad news.

\section{COMPARATIVE ANALYSIS}

The following sections discuss some characteristics of the studied protocols that could affect energy consumption.

Indeed, some significant differences, a point of view of energy consumption between the proactive and reactive behavior of routing algorithms studied can be identified to facilitate the understanding of the simulation results: 
International Journal of Computer Networks \& Communications (IJCNC) Vol.6, No.3, May 2014

- Usage of bandwidth: the choice between on demand or reactive routing protocol in terms of consumption of energy, the fact that one must consume energy to update periodically route table and in the other case, it must consume energy only to discover that a route where communication must be established. In protocols proactive routing, many route discovered by standing broadcasts control packets roads will never be used and the bandwidth consumed for these discovery is wasted. Clearly, the reactive protocols have an advantage on this point (but probably not in delay for the routing).

- Management of invalid or congested routes : AODV seems better able to monitor more finely invalid paths since it uses the timer expirations route those serving regular routes considered too ancient and force for new discoveries of routes (sometimes unnecessarily). In addition, these messages "Hello" are used to verify the validity of links (but these control packets that evoke moreover entirely those used in the protocols pro-active energy-consuming, even for a link that not used then).

The previous elements can be summarized with the following table:

Table 1. Comparisons of the characteristics of the routing protocols.

\begin{tabular}{|l|l|l|l|}
\cline { 2 - 5 } \multicolumn{1}{c|}{} & \multicolumn{1}{c|}{ AODV } & \multicolumn{1}{c|}{ DSDV } & \multicolumn{1}{c|}{ AOMDV } \\
\hline Routing categories & Reactive routing & Proactive routing & Reactive routing \\
\hline Route maintained in & Routing table & Routing table & Routing table \\
\hline Discovery of necessary route & Yes & No & Yes \\
\hline Necessary periodic update & No & Yes & No \\
\hline Update from & No update & All the neighbours & No update \\
\hline Uses "Hello" message & $\begin{array}{l}\text { Yes ( actifs } \\
\text { neighbors) }\end{array}$ & Yes & Yes \\
\hline $\begin{array}{l}\text { Route inserted into the } \\
\text { header of the packet }\end{array}$ & No & No & No \\
\hline Uses timer route & Yes & No & Yes \\
\hline Multiple route available & No & No & Yes \\
\hline
\end{tabular}

So each approach has its own advantages and disadvantages that we can evaluate via simulations to understand exactly how they work in relation to energy use.

\subsection{Modelling Scenarios in NS2}

NS-2 [13] is an open source discrete event simulation tool to explore the performance of wired and wireless networks in terms of underlying protocol stacks, routing algorithms, network traffic, etc. The primary function of NS-2 is to provide a network of nodes which are able to communicate together with the other by sending or receiving of the data packets on each network, and eventually generate the traffic traces network for more analysis. To achieve this, NS-2 
contains a great diversity of network related components that can be flexibly assembled by a descriptive language called Tool Command Language (Tcl) specific to certain networking configurations to allow different simulation scenarios.

\subsection{The Energy Model Used In NS-2}

The NS-2 extension comprises an energy model that advises every node about its instantaneous energy level. For using this model, we need to set three parameters: the initial energy (InitialEnergy), the transmission power (txPower) and reception power (rxPower). These two last values, Multiplied by the duration of transmission or reception of a packet, provide respectively the quantity of energy required for the transmission or the reception of a packet. In our simulation study, we have fixed these parameters to the following values:

Table 1. Parameter of Energy Model.

\begin{tabular}{|l|l|}
\hline \multicolumn{1}{|c|}{ Parameter } & \multicolumn{1}{c|}{ Value } \\
\hline Network Interface & WirelessPhy \\
\hline MAC Type & 802.11 \\
\hline Channel & WirelessChannel \\
\hline Propagation & TwoRayGround \\
\hline Antenna & OmniAntenna \\
\hline Queue & DropTail/PriQueue \\
\hline Initial Energy & 100 joule \\
\hline Reception Power & $35.28 \mathrm{~mW}$ \\
\hline Transmission Power & $31.32 \mathrm{~mW}$ \\
\hline
\end{tabular}

We attributed to each node an initial energy of 100 joules to be diminished as the node transmits or receives packets. If the energy level of a node drops to zero, it is considered as a dead node, i.e. it is no longer able to take part in communications. It is known that the energy consumption of a node is primarily due to the transmission and the reception of data or controlling packets (such as RREQ, RREP, RERR, HELLO). In order to measure this amount of energy consumed during the transmission process (noted txEnergy), we should multiply the transmission power (txPower) by the time needed to transmit a packet:

\section{txEnergy $=$ txPower $x($ packetsize/bandwidth $)$}

And for a received packet:

rxEnergy $=$ rxPower $\mathbf{x}($ packetsize/bandwidth $)$

In this study, we used the network simulator NS-2.35 to compare the three routing protocols (AODV, AOMDV and DSDV). The wireless mesh network consists of $2 \mathrm{GW}$ (Gateways), $6 \mathrm{MR}$ (Mesh Routers) and 2 Mesh Clients (Mobile nodes) which are distributed a 500m X 500m area as basic scenario. The initial energy of each node is taken as $100 \mathrm{~J}$ with transmission and receiving 
power consumption. The simulation model with parameters is listed in table 1 . The data packet size is of 512 bytes. Traffic model used is CBR (Constant Bit Rate). CBR Model generates traffic at a constant rate of 4 packets per second.

\subsection{Results and Analysis}

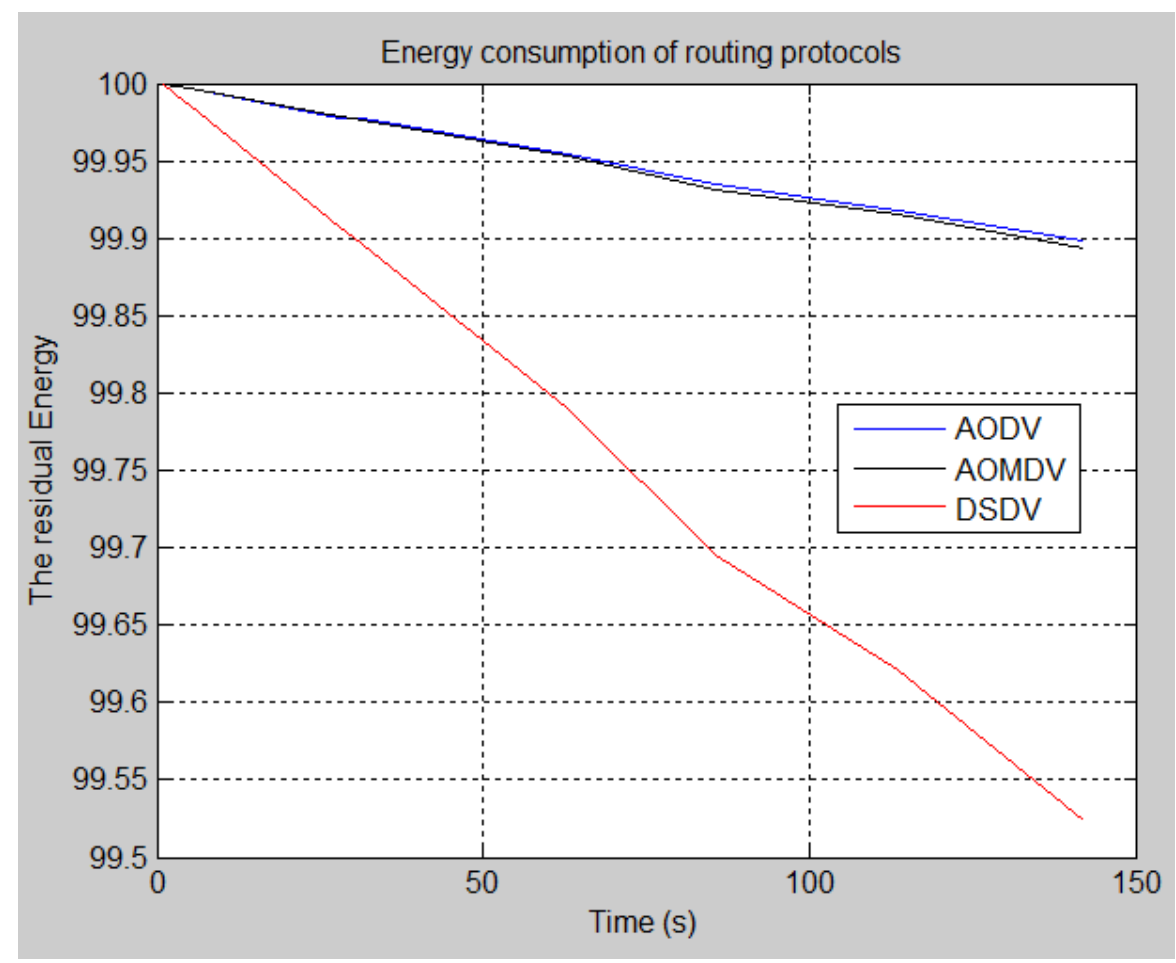

Figure 2. Energy consumption of routing protocols

In Figure 2, we can see that the residual energy (remaining energy) of routing protocols AODV, AOMDV and DSDV decreases as time increases. However, there is a difference among proactive and reactive routing protocols. In the proactive routing protocol all nodes updated each other on the same network and make update their tables away to maintain a consistent approach and view network updates, that result in an energy consumption but with the reactive protocol when a node requires a route to its destination, it initiates a route discovery process within the network. The process is over when a route lies or every possible permutation of the route has been reviewed. Simulation results show that the AODV and AOMDV routing protocol consumes less energy than the DSDV routing protocol.

\section{ROUTING PROTOCOLS OVER A DISTRIBUTED TDMA MAC PROTOCOL}

In this section, we propose a Time Division Multiple Access (TDMA) protocol to schedule flows of traffic in slots. Scheduling the flows can reduce energy waste.

\subsection{Methodology}

A Time Division Multiple Access (TDMA) Medium Access Control (MAC) protocol [14] that coordinates the delivery of data to receivers based on the gateways control. 
International Journal of Computer Networks \& Communications (IJCNC) Vol.6, No.3, May 2014

There are three steps in this TDMA: up-link step into which nodes transmit data to the base station, down-link step where the base station transmits data to the nodes, and reservation step wherein nodes request new connections. The base station dictates a frame structure within its range. A frame made up a number of data and a traffic control. Nodes with steady traffic are indicated in a list, which enables nodes with no traffic to quickly reduce power. The traffic control is transmitted by the base station and contains information about the subsequent data, including when the next traffic control will be transmitted. Nodes explicitly request transmission from the base station, in a distributed manner, during the reservation phase. In our approach, the gateway performs the slot assignment based on its routing decisions.

To further improve the energy efficiency, according to wireless mesh network, this paper design energy-aware TDMA routing protocols based MAC layer whose slot assignment is managed by the gateway. The gateway informs each node about slots in which it should listen to other nodes' transmission and about the slots, which the node can use for its own transmission. The advantages of using a TDMA MAC layer are:

- TDMA protocol builds clock synchronization to refresh the energy model and send rerouting decision from the gateway to the nodes.

- The collision between the nodes can be avoided, because each node has its own assigned time slots.

Additional problems can happen with the existence of communication errors: a packet that contains the slot assignment can be dropped. Whether a node that does not learn the decision of the gateway turns off itself and no collisions can happen. However, we prefer to make up another alternative that a node preserves its previous state, if it does not get a packet routing from the gateway in the predetermined time slot, leading in potential collisions. However, the probability of collision is limited for the reasons acquired in [15].

There are some inconvenient of a TDMA protocol against a random access protocol, such as IEEE 802.11 [16]. First, the scheduling must be made by predicting the arrival of future traffic, delaying packets until the scheduling can be done or asking traffic to track a predictable pattern. Also, TDMA requires synchronization to divide the time in slots over the entire network.

\subsection{Integration issues and performance evaluation}

In this evaluation, we used the network simulator NS-2 to compare the AODV and AOMDV routing protocols using the design of TDMA MAC layer and a conventional MAC layer. The network contains 2 GW (Gateways), 6 MR (Mesh Routers) and 2 Mesh Clients (Mobile nodes) distributed in a 500m X 500m area as basic scenario. Traffic model used is CBR; the packet size is 512 bytes packet rate is 4 packets per second. In NS 2, two MAC layer protocols are implemented, which are 802.11 and TDMA. In this section we briefly discuss TDMA MAC protocol.

In contrast contention based MAC protocol (802.11, for example), a TDMA MAC protocol allows different time slots for nodes to send and receive packets. The superset of these time slots is called a TDMA frame which contains preamble besides the data transmission slots as shown in Figure.3. Within the preamble, every node has a dedicated sub-slot and uses it to broadcast the destination node id of outgoing packet. Other nodes listen in the preamble and saved the time slots to receive packets. Each node has a data transmission slot to send packets [17]. 
International Journal of Computer Networks \& Communications (IJCNC) Vol.6, No.3, May 2014

Frames 0-11:TCH Frame 12:SACCH Frames 13-24:TCH Frame 25: Unused

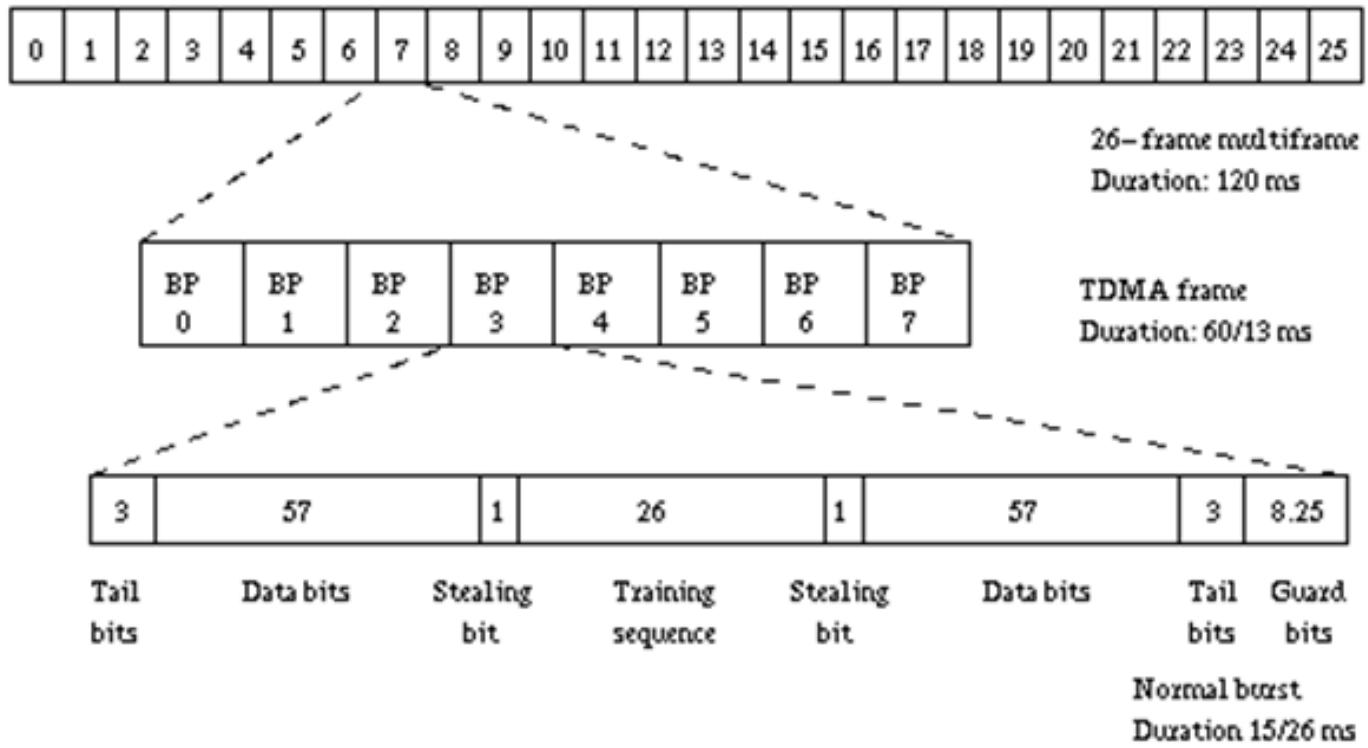

Figure 3. An example of TDMA frame structure

To prevent unnecessary power consumption, each node turns its radio on and off explicitly by invoking node API set_node_sleep().

The radio may be on when: in the preamble phase (takes one slot time) and there is a packet to send and receive. The preamble is implemented as a central data structure TDMA_preamble, that is accessible to all the nodes. At the start of a frame, each node writes the destination node id into its sub-slot in preamble if it has a packet to send. After the preamble phase, each node sends packet in its data transmission slot and checks the preamble to identify if there is a packet to receive in other slots.

Figure 4, depicts a comparison between the routing protocols based 802.11 MAC protocols (called AODV, AOMDV) and energy-aware routing protocols TDMA based MAC protocol so called TDMA-AODV and TDMA-AOMDV function of time simulation. We can say that the TDMA-AODV approach provides more interesting results in residual energy ration which consume less energy than the AODV based 802.11 MAC. TDMA based protocols are more energy efficient, and the energy consumed is proportional to the duration of the transmission cycle whereas the latency is proportional to the size of the network.

The TDMA-AODV seems to be a more promising solution, which has the natural advantages of having no contention introduced overhead and collisions. Furthermore, TDMA can guarantee a deterministic delay bound. 
International Journal of Computer Networks \& Communications (IJCNC) Vol.6, No.3, May 2014

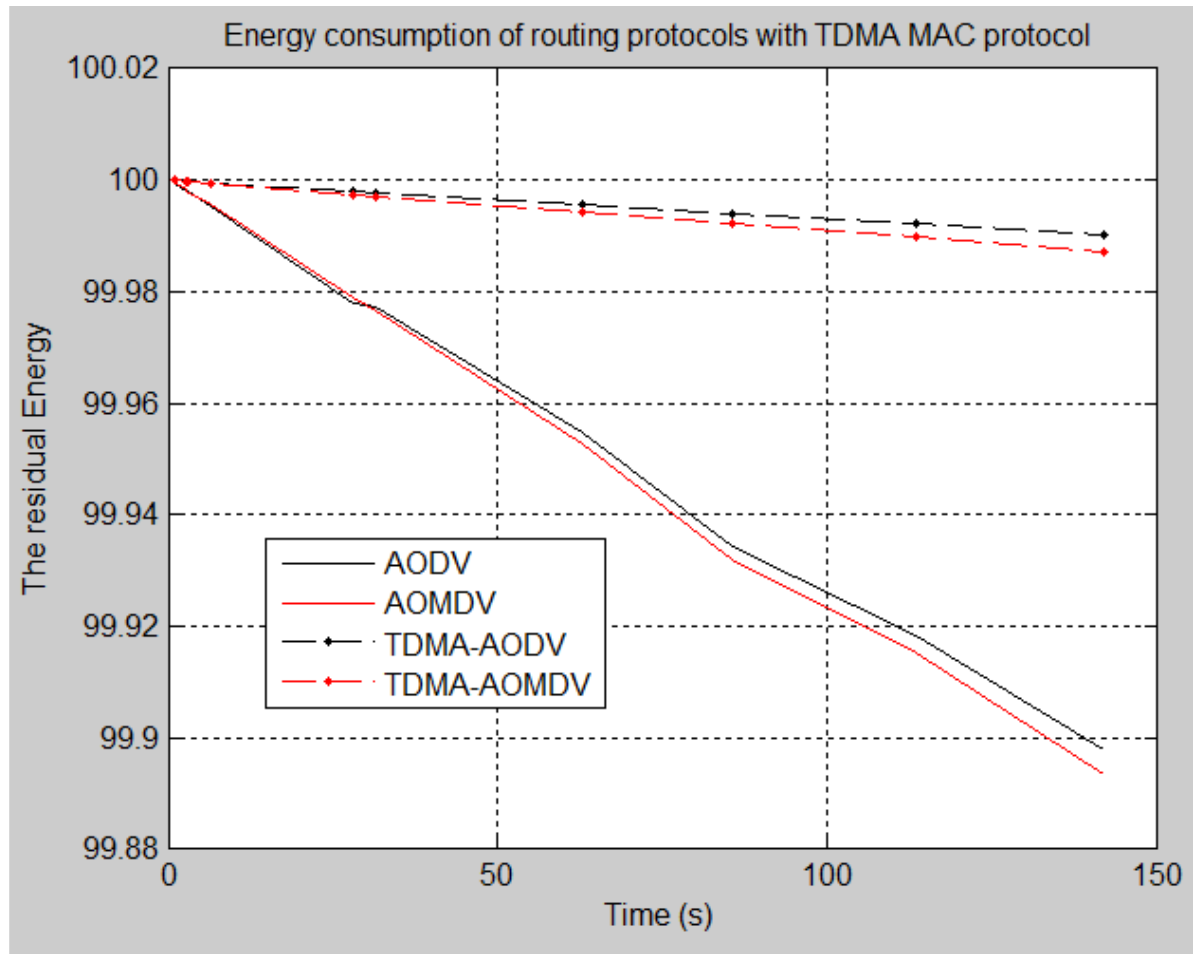

Figure 4. Energy consumption of routing protocols with TDMA MAC protocol

Owing to its benefits reduced collisions, scalability and latency limited, TDMA is widely regarded in wireless networks. TDMA partitions time into many fixed slots and nodes transmit data in their assigned slots, thereby avoiding collisions.

\section{CONCLUSION}

In this paper, we introduced a novel approach for energy aware routing based on TDMA MAC protocol for wireless mesh networks. First, we reviewed the existing routing protocols and we compared them with the characteristics of energy consumption. AODV routing protocol consumes less energy compared with AOMDV and DSDV. After, we focus on the design of routing protocols that are implemented in TDMA based MAC cross-layer wireless mesh networks. Owing to its advantages of reducing collisions, TDMA-based protocols are more energy efficient. However, TDMA-AODV approach provides more interesting results in residual energy ration compared with the AODV based 802.11 MAC. TDMA based protocols are more energy efficient, and the energy consumed is proportional to the length of the transmission cycle while the latency is proportional to the size of the network. TDMA-AODV seems to be a promising solution. 
International Journal of Computer Networks \& Communications (IJCNC) Vol.6, No.3, May 2014

\section{REFERENCES}

[1] Yan Zhang, Jijun Luo and Honglin Hu, Wireless Mesh Networking: Architectures, Protocols and Standards (Wireless Networks and Mobile Communications), December 13, 2006 I ISBN-10: 0849373999.

[2] I.F. Akyildiz and X. Wang, (2005) “A survey on wireless mesh networks". IEEE communications Magazine 43(9), s23-s30.

[3] A. Bianzino, C. Chaudet, D. Rossi and J. Rougier, -A Survey of Green Networking Research $\square$, Communications Surveys \& Tutorials, IEEE, décembre 2010 .

[4] S.Mamechaoui, F.Didi and G.Pujolle, A Survey On Energy Efficiency For Wireless Mesh Network , International Journal of Computer Networks \& Communications (IJCNC) Vol.5, No.2, March 2013.

[5] Microsoft Mesh Networks. Available at: http://research.microsoft.com/mesh/.

[6] Routing Protocol for Mobile Ad hoc Networks (DSR),' IETF Internet Draft,draft-ietf-manet-dsr10.txt, July 2004, work in progress.

[7] Kiyon Autonomous Networks. Available at: http://www.kiyon.com.

[8] Charles E. Perkins, Elizabeth M. Belding-Royer, and Samir R. Das, "Ad hoc On-Demand Distance Vector (AODV) Routing,’ IETF Experimental RFC 3561, July 2003.

[9] Sikander Singh, Dr. Trilok Chand Aseri, Scalable Energy Efficient Ad-Hoc On Demand Distance Vector See-Aodv Routing Protocol In Wireless Mesh Networks - Ubiquitous Computing and Communication Journal, 2011.

[10] Ashok Kumar Sharma and Supratik Banerjee, Route Discovery Utilizing Power in AODV, International Journal of Computer Trends and Technology- volume4Issue 1- 2013.

[11] Patil, V. C., Biradar, R. V., Mudholkar, R. R. and Sawant, S. R. On-Demand Multipath Routing Protocols for Mobile Ad-Hoc Networks Issues and Comparison. International Journal of Wireless Communication and Simulation 2 (2010) 21-38.

[12] A.H.Abd Rahman, Z.A.Zukarnain,"Performance in Comparison of AODV, DSDV and I-DSDV Routing Protocols in Mobile Ad Hoc Networks", European Journal of Scientific Research Vol.31, No. 4, pp. 556-576 (June 2009).

[13] www.isi.edu/nsnam/ns/ Network simulator ns-2.

[14] P. Havinga, G. Smit, "Energy-efficient TDMA medium access control protocol scheduling", Proceedings Asian International Mobile Computing Conference (AMOC 2000), Nov. 2000.

[15] Arisha, A. Youssef and F. Younis, Energy-Aware TDMA-Based MAC for Sensor Networks, IEEE Workshop on Integrated Management of Power Aware Communications, Computing and Networking IMPACCT 2002.

[16] Wireless LAN medium access control (MAC) and physical layer (PHY) specifications, IEEE Standard 802.11, June 2007.

[17] Kevin Fall, Kannan Varadhan, The ns Manual (formerly ns Notes and Documentation), March 14, 2008. See http://www.isi.edu/nsnam/ns/ns-documentation.html 